\title{
Flow Markers in Microreactors: A Generally Applicable Chroma- tographic Method for Monitoring Flow Rates During Reactions
}

\author{
Pieter J. Nieuwland, Kaspar Koch, Jan C.M. van Hest and Floris P.J.T. Rutjes* \\ Institute for Molecules and Materials, Radboud University Nijmegen, Heyendaalseweg 135, 6525 AJ Nijmegen, \\ The Netherlands
}

\begin{abstract}
Microreactors have found widespread use for continuous flow synthesis and reaction optimization. Flow rates are critical factors with respect to the latter application because they are used to set screening parameters such as reaction time and stoichiometric ratios. However, the set flow values of pumps for nanoliter to microliter volume reactors are quite often not sufficiently accurate. In this paper we present a generally applicable chromatographic method to analyze flow rates during microreactor reaction screening. By adding flow markers to all reactant and reagent flows and performing conventional GC analysis on all samples, an accurate flow rate was calculated. The deviation between the set flow values and the measured flow rates was shown for a standard continuous flow experiment. The implications of this deviation for reaction optimization were demonstrated via a model Swern-Moffatt oxidation reaction, which showed that accurately measured flow rates are critical for correct data interpretation.
\end{abstract}

Keywords: Flow chemistry, micoreactors, flow markers, flow rate, parametric optimization, oxidation.

\section{INTRODUCTION}

The interest in using microreactors for synthesis has increased enormously in recent years [1-6]. Traditionally, the emphasis has either been on the production of chemicals in microstructured flow reactors, which has several benefits over conventional batch reactions, or on rather specialized and novel reaction processes on a very small scale [7, 8]. Scaling up, or scaling out using microreactor setup multiplication, has been a particular subject of investigation [9]. Surprisingly, few examples exist on the application of microliter or nanoliter volume reactors for screening purposes, in particular for reactions that are commonly used in synthesis laboratories [10].

Several experiments involving pressure-driven flow in low volume microreactors have been reported [8, 11-16]. Most setups consist of syringes loaded on syringe pumps and connected to a microreactor via tubing. This leads to a continuous flow system, i.e. reactants and reagents are continuously pumped into the microreactor and a stable outflow of starting material, products and possibly by-products is obtained. Typically, the flow rates in combination with the reactor's internal volume determine parameters such as reaction time and reagent stoichiometry. Since these parameters are often the subject of the investigation (e.g. in the optimization of a reaction), it is crucial to exactly control and know the flow rates.

In most studies that have been published, it is not clear how flow rates were measured; most probably they were based on the set flow values. We recently carried out several reactions using a pressure-driven microreactor system with

*Address correspondence to these authors at the Institute for Molecules and Materials, Radboud University Nijmegen, Heyendaalseweg 135, 6525 AJ Nijmegen, The Netherlands; Tel: +31 24365 3202; Fax: +31 24365 3393; E-mail: f.rutjes@science.ru.nl syringes and syringe pumps, and found that these pump settings are not reliable. Therefore we created a protocol to measure the average flow rate during each experiment, resulting in intrinsically reliable flow data. As a result, the observed data can be reproduced on other micro systems, which currently is often not possible. Once the exact optimal parameters are found, the reaction can be directly translated into either larger scale continuous flow systems or into conventional preparative synthesis. In this paper we present this protocol applied to our system. A case study on reaction optimization is included that clearly illustrates the discrepancy in results that otherwise might exist, demonstrating the importance of being able to accurately determine flow rates.

Possible causes of flow rates deviating from set flows are lack of calibration of the syringe pump itself, high back pressure built up in the system, syringes suffering from static and dynamic friction (causing jerky motion of the syringes' plungers), undetectable leaking of connectors, etc.

In principle, very low flow rates typically required for our experiments, down to $0.5 \mu \mathrm{L} / \mathrm{min}$, could be continuously monitored either by using flow meters or externally using micro Particle Image Velocimetry ( $\mu$ PIV). These methods, however, present the experimenter with some challenges. All available microflow meters use the thermal principle. However, these flow meters must be calibrated according to the thermal properties and thus chemical composition. This is not practical for reaction optimization because compositions rapidly change between experiments and even within experiments. Furthermore, $\mu$ PIV cannot be regarded as a generally applicable method for synthesis monitoring because extensive optical equipment and typically fluorescent particles are required [17].

Some techniques offer real-time information concerning the results from the outflow, while others have a time delay between performing a reaction and receiving the informa- 
tion. Real-time techniques include UV/Vis spectroscopy [1820], infrared spectroscopy [21], Raman spectroscopy [22, 23] and Thermal Lens Microscopy [24-26]. Although having the benefit of a quick response to changing parameters, these techniques are generally quite limited in selectively detecting compounds in a generic way. Chromatographic methods such as conventional HPLC-UV and GC-FID can be much more generally applied to most synthesis reactions for quantitative determination of reaction yield, conversion and selectivity [12].

During our reaction optimization experiments we efficiently acquire chromatographic data for each sample through standard automated methods. We reasoned that these analyses could easily be combined with flow rate monitoring. When a certain 'flow marker', i.e. an internal standard, is added to each fluid and another internal standard is added to the dilution fluid in the collection samples, the average flow rate can readily be calculated by chromatographic ratios and calibration curves (Fig. 1). In eqn (1), $Q_{A}$, the flow rate of fluid $A$, is calculated. $\left[\mathrm{M}_{\mathrm{A}}\right]$ is the concentration of the flow marker in the measured sample, while $\left[\mathrm{M}_{\mathrm{A}}\right]_{0}$ is the known starting concentration of the flow marker in fluid A before it enters the microreactor. $\mathrm{V}_{\mathrm{dil}}$ is the known volume of dilution fluid and $t_{\text {coll }}$ is the known collection time of the sample (a derivation of eqn (1) can be found in S1 of the supporting information).

$Q_{A}=\frac{\left[M_{A}\right] V_{d i l}}{t_{\text {coll }}\left(\left[M_{A}\right]_{0}-\left[M_{A}\right]\right)}$

A careful selection of the flow markers is required: they must not interfere with the chemical reaction and should be adequately separated by HPLC or GC (Fig. S3 in supporting information). In order to evaluate the suitability of internal standard candidates, retention times using the current GC method were determined, and any candidates interfering with other analytes were excluded. Subsequently, the candidates were examined on reaction interference by analyzing reaction mixtures with and without the candidates. Due to the chromatographic separation, accurate quantification is easily realized. Because the method is integrated into existing chromatographic analysis, flow rate data is obtained with very little effort. In order to quickly and efficiently select correct flow markers, retention time libraries can be used.

\section{MATERIALS AND METHODS}

\section{Gas Chromatography}

GC analysis was performed on a Shimadzu GC 2010 GCFID equipped with a Quadrex 0071701 column (length: 10 $\mathrm{m}$, internal diameter: $0.1 \mathrm{~mm}$, film thickness: $0.1 \mu \mathrm{m})$, using a temperature program starting at $98{ }^{\circ} \mathrm{C}$ for $0.85 \mathrm{~min}$ with subsequent ballistic heating with a set temperature of $235^{\circ} \mathrm{C}$ for $1.0 \mathrm{~min}$, a linear flow rate of $1 \mathrm{~m} / \mathrm{s}$ and a split ratio of 750. The sample concentration and split ratio led to a column loading exceeding the limit of detection in a factor of at least 100. An analysis cycle time of approximately 3 min was used. For GC calibration, the internal standard method was used, and in each sample, an additional internal standard with a fixed concentration was present. For this internal standard, 1-bromonaphthalene was used in a concentration of $0.2 \% \mathrm{v} / \mathrm{v}$.

\section{Microreactor Setup [15]}

All syringes (Harvard apparatus; high pressure syringe, 2 $\mathrm{mL}$ ) mounted on a syringe pump (New Era; type NE-1000 or NE-500) were connected to FEP tubing (1.59 mm OD, 254 $\mu \mathrm{m}$ ID). At the end of each piece of tubing, a special 'flat bottom headless nut' (Upchurch Scientific; type: M-660) was mounted which pressed down onto a flat bottom ferrule (Upchurch Scientific; type: M-650) to achieve a leak free fluid connection to the microreactor. The microreactor was placed in a custom-designed chip holder with threaded holes on the top side in which the nuts were screwed. A stainless steel needle (UpChurch Scientific; type U-106 1/100" ID $1 / 16$ " OD, custom shaped needle tip) was used as the outlet. A sample robot (Gilson Aspec XL) with dedicated software (Gilson 735 Sample software, Version 1.00) was used to dispense all samples during reaction screening. The pumps were programmed with a script running in ZOC Terminal V 5.0 in order to control the flow speed of the three pumps. Furthermore, the script provided switching events for the robot to automatically control stabilization time and collection time.

\section{Microreactor}

The microreactor was fabricated from borosilicate glass by Micronit Microfluidics BV (Enschede, The Netherlands) (HF etched). Chip dimensions are given in Table 1. From these dimensions, combined with the flow rates and viscosi-

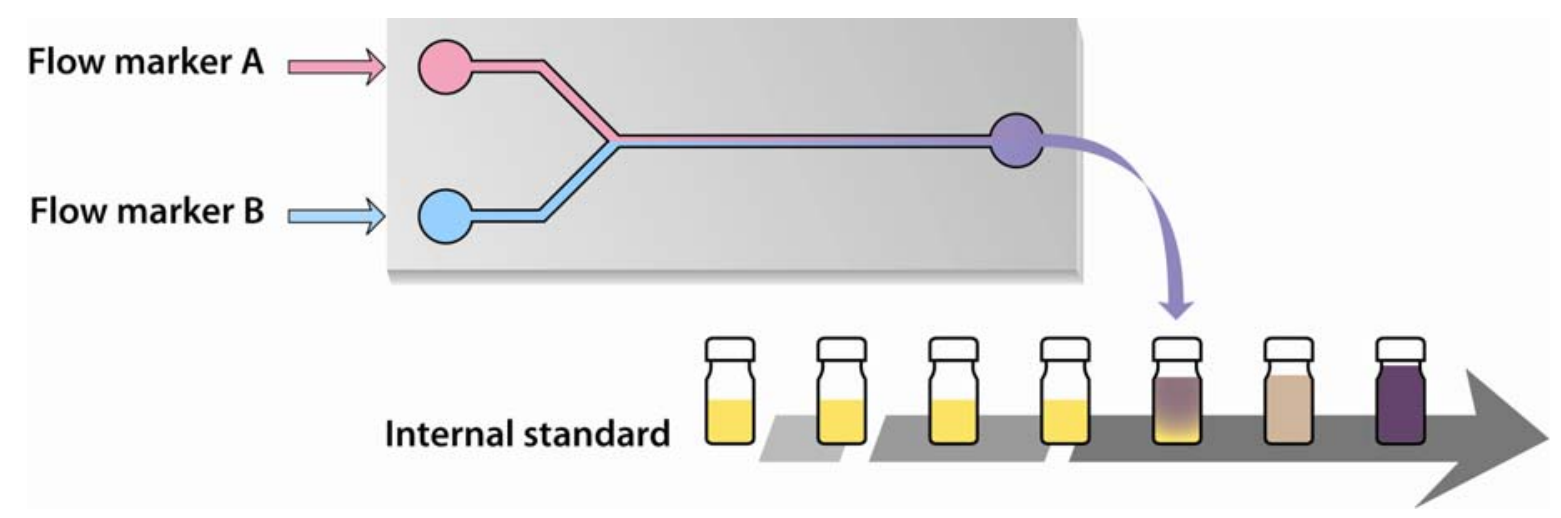

Fig. (1). The concept of flow markers. Two fluids containing a known concentration of different flow markers are continuously pumped through the microreactor with flow rates that determine reaction time and stoichiometry. When mixed with an internal standard in the collecting vial, the amount of flow marker and thus the flow rates can be accurately measured. 
ties employed in the experiments, it can be expected that all experiments are within the laminar flow regime. The channel layout included two T-junction mixers placed in series (the channel design is provided in the supporting information, S2).

Table 1. Microchannel Dimensions

\begin{tabular}{|c|c|}
\hline Total length microchannel & $29.8 \mathrm{~mm}$ \\
\hline Depth & $58 \mu \mathrm{m}$ \\
\hline Width $^{\text {a }}$ & $114 \mu \mathrm{m}$ \\
\hline Internal reaction volume $^{142 \mathrm{~nL}}$ \\
\hline
\end{tabular}

${ }^{a}$ Width at the top of the channel. Channel cross section has a standard wet etch geometry.

\section{Flow Measurement Experiment}

GC was used for flow rate measurement. Two solutions consisting of a solvent (dichloromethane) and a flow marker (1,2-dichlorobenzene for fluid A and 5-bromo-m-xylene for fluid B, both approximately $2 \% \mathrm{v} / \mathrm{v}$ ) were pumped into the mixing chip. One of the two outflow channels was redundant and thus blocked. The effluent was collected in standard 1.5 $\mathrm{mL}$ vials. The vials were prefilled with $1000 \mu \mathrm{L}$ of a solution of 1-bromonaphthalene as the internal standard in dichloromethane $(0.2 \% \mathrm{v} / \mathrm{v})$. The syringes were loaded with the solutions and all fluidic connections were established. The outflow needle of the chip was then placed into the waste container and the pumps were started. After a predetermined stabilization time, the outflow needle was moved to the first collection vial. More vials were filled when duplicate or triplicate measurements were carried out. Then the outflow nee- dle was moved back to the waste container and flow settings were changed. After stabilization the next set of samples was collected and the procedure was repeated until all flow conditions were screened. Table 2 shows a selection from the total experimental setup.

\section{Swern-Moffatt Model Reaction[27, 28]}

GC was used for flow rate measurement and aldehyde yield determination. A solution of benzyl alcohol (2, $0.5 \mathrm{M})$, DMSO $(2.5 \mathrm{M})$ and dichlorobenzene as a flow marker $(2 \%$ $\mathrm{v} / \mathrm{v}$ ) in dichloromethane (solution $\mathrm{A}$ ) and a solution of trifluoroacetic anhydride (TFAA) $(0.5 \mathrm{M})$ in dichloromethane with mesitylene as a flow marker $(2 \% \mathrm{v} / \mathrm{v})$ (solution B) were pumped into the mixing chip. To finish the reaction in the microreactor, pure $\mathrm{N}, \mathrm{N}$-diisopropylethylamine (DIPEA) was pumped into the reactor. The experiment was carried out as described in the previous section. The flow rate, collection times and waste times, during which the continuous flows are stabilized, are listed in Table $\mathbf{2}$.

\section{RESULTS AND DISCUSSION}

In order to evaluate the flow rate accuracy of the microfluidic system, an extensive flow test experiment was carried out. Two syringe pumps connected to a simple mixing chip delivered stock solutions of flow markers. The flow rates of the pumps were varied according to the values depicted in Table 2, while samples were being taken for an exact amount of time, also listed in Table 2 . In order to simulate flow parameters in a real reaction kinetics experiment, the set flow rates were calculated from linear arrays of ratios (typically determining reagent stoichiometry) and residence times. Both parameters were sampled at exponential intervals.

Table 2. A Selection from the Experimental Setup for the Flow Measurement Experiment ${ }^{\mathrm{a}}$

\begin{tabular}{|c|c|c|c|c|}
\hline Set flow A $(\mu \mathrm{L} / \mathrm{min})$ & Set flow $B(\mu \mathrm{L} / \mathrm{min})$ & Residence time (min) & Waste time $(\min )^{b}$ & Sampling time (min) \\
\hline \multicolumn{5}{|c|}{ Flow measurement experiment ${ }^{\mathrm{c}}$} \\
\hline 3.25 & 1.62 & 0.977 & 12.3 & 15.39 \\
\hline 52.22 & 47.8 & 0.048 & 0.42 & 0.96 \\
\hline 4.66 & 4.26 & 0.534 & 4.69 & 10.74 \\
\hline 2.54 & 2.33 & 0.977 & 8.59 & 19.65 \\
\hline \multicolumn{5}{|l|}{ Swern-Moffatt oxidation $^{\mathrm{d}}$} \\
\hline 16.20 & 37.27 & 4.69 & 1.85 & 3.09 \\
\hline 112.6 & 146.4 & 0.97 & 0.27 & 0.440 \\
\hline 65.41 & 52.33 & 2.13 & 0.57 & 0.76 \\
\hline 12.73 & 40.74 & 4.69 & 2.36 & 3.93 \\
\hline
\end{tabular}

${ }^{a}$ The complete data set can be found in S3 and S4 of the Supporting Information. ${ }^{\mathrm{b}} \mathrm{After}$ setting the appropriate flow rate, the outflow needle was placed in a waste container. ${ }^{\mathrm{c}} \mathrm{Solu}-$ tion of flow markers A and B, respectively, in $\mathrm{CH}_{2} \mathrm{Cl}_{2}(2 \% \mathrm{v} / \mathrm{v}) .{ }^{\mathrm{d}}$ For flow A: Solution of benzyl alcohol $(0.5 \mathrm{M})$, DMSO $(2.5 \mathrm{M})$ and a flow marker A $(2 \% \mathrm{v} / \mathrm{v})$ in $\mathrm{CH}_{2} \mathrm{Cl}_{2}$. For flow B: Solution of TFAA $(0.5 \mathrm{M})$ and flow marker B $(2 \% \mathrm{v} / \mathrm{v})$ in $\mathrm{CH}_{2} \mathrm{Cl}_{2}$. 
Then, the concentration of the flow markers relative to the concentration of the internal standard was determined by GC-FID, and the amount of both fluid A and B collected into the sample vials was calculated. Finally, using the known internal volume of the microreactor, the actual flow rates were determined. Because this method calculates the average flow rate over the time of sample collection this data can directly be used to calculate residence times and stoichiometric data.

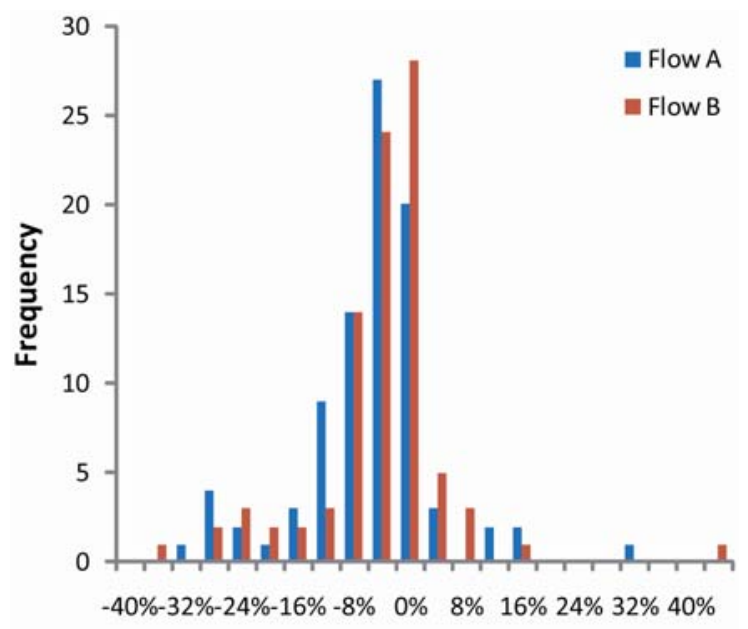

Flow deviation

Fig. (2). Histogram of flow deviations of the flow check experiment for both flow $\mathrm{A}$ and $\mathrm{B}$.

Fig. (2) shows the results as a histogram plot. It is clear that the majority of screening points have a minor deviation, but there are data points at which the actual flow rate is quite different from the set flow rate. The differences between the measured flow rate and the flow rate set into the pump can also be shown as a vector plot (Fig. S4 in the supporting information). The average differences are $-7.5 \%$ for flow $\mathrm{A}$ and $-6.3 \%$ for flow B, with standard deviations of 9.6 and $9.7 \%$, respectively, indicating a significant systematic error. The histogram shows a relatively narrow distribution, with most data points between -12 and $+4 \%$ deviation, however, there are some outliers present.

The relative errors of this method must be brought into consideration. Typically, measurement inaccuracy consists of errors in the volume of collection fluid, sampling time and calibration of the GC method, resulting in a typical error value of $2 \%$ relative to the calculated flow rate. Therefore, flow deviations discussed above can be regarded significant.

From this validation experiment it is evident that it is absolutely vital to monitor the flow rates of reactants and reagents in microreactor flow experiments, especially since they imply an important input variable in the experiment.

Now that it was established that flow monitoring is necessary, we decided to evaluate flow deviations in a chemical synthesis experiment. The Swern-Moffatt oxidation (Fig. 3) was chosen as a model reaction. Yoshida et al. previously showed in an elegant way that this oxidation can be performed in a microreactor at room temperature conditions [28]. Additionally, an extensive optimization of this particular reaction study was performed by the authors [27]. Crucial in this microreactor process is a fast reaction of the reactive intermediate $\mathbf{2}$ with benzyl alcohol $\mathbf{1}$ in order to circumvent the undesired Pummerer rearrangement leading to byproducts 6 and 7.
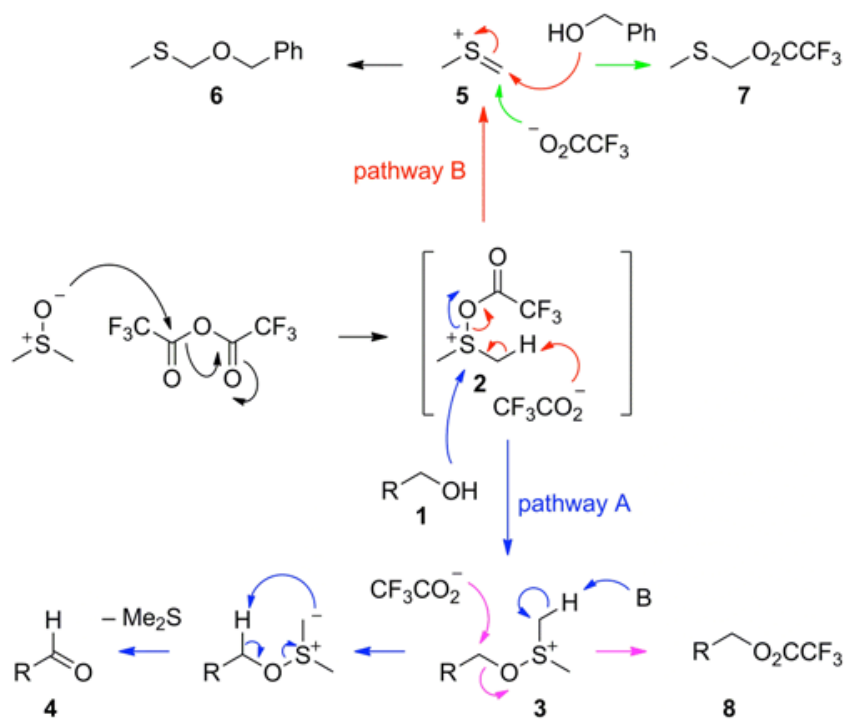

Fig. (3). Swern-Moffatt oxidation of benzyl alcohol.

In our approach, benzyl alcohol was premixed with dimethyl sulfoxide (DMSO), prior to reaction with trifluoroacetic anhydride (TFAA) [29-31] while DIPEA was added at the end of the channel, performing the final elimination step in the reaction and quenching the excess of TFAA at the same time (Fig. 4). To both solutions A and B inert flow markers were added. The flow marker in solution $B$ also served as an internal standard for the reaction, to which peak intensities of starting material and products could be related.

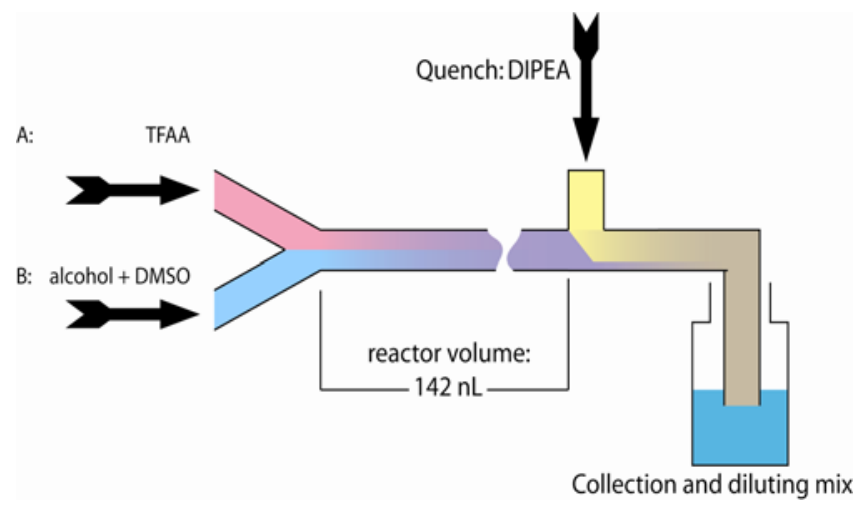

Fig. (4). Flow scheme of microreactor system for Swern-Moffatt oxidation.

Table 3. Averages and Standard Deviation in Flow Offsets

\begin{tabular}{|c|c|c|}
\hline Flow & Average Offset & Standard Deviation in Offset \\
\hline \hline A & $-11.7 \%$ & $7.4 \%$ \\
\hline B & $13.8 \%$ & $6.9 \%$ \\
\hline
\end{tabular}

The flow rates of flow $\mathrm{A}$ and $\mathrm{B}$ were varied in such a way that these settings would result in a matrix of data points with different retention times and stoichiometries (Table $\mathbf{2}$, lower part). Distributions similar to the flow measurement 


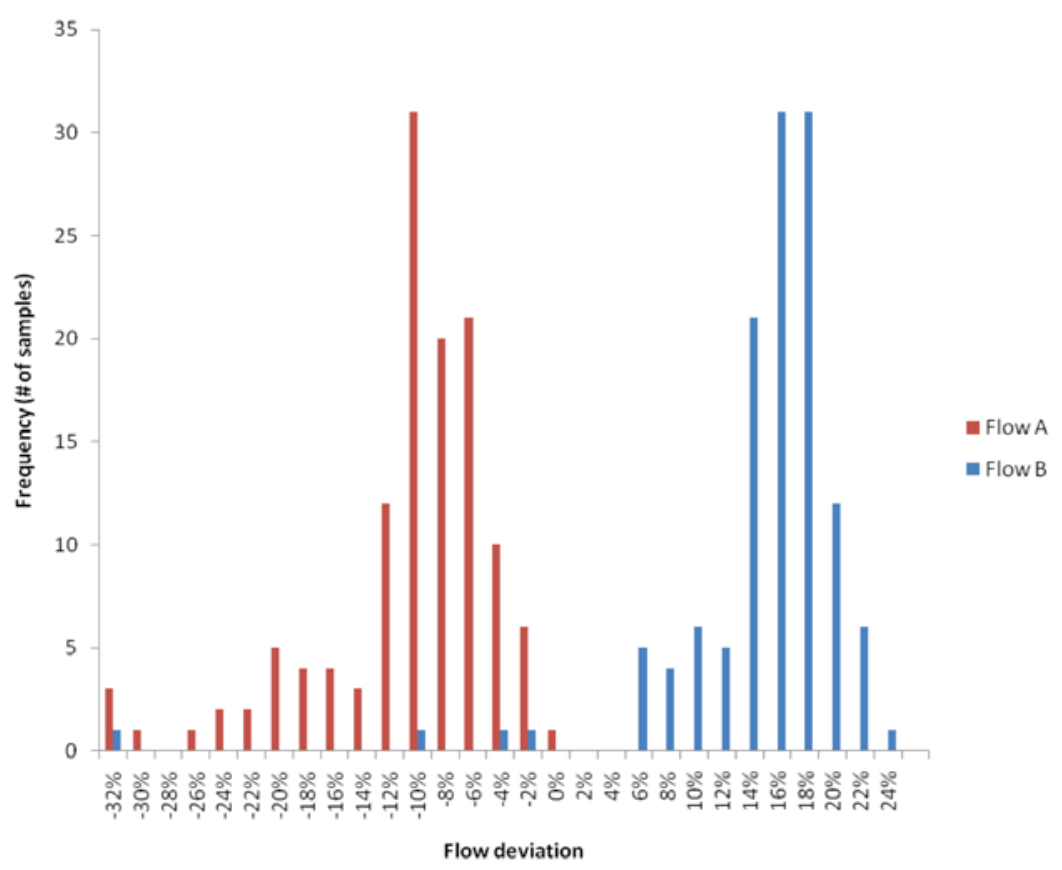

Fig. (5). Histogram of flow deviations of the Swern oxidation experiment for both flow A and B.

experiment were observed, but the average offsets were higher (Table 3). The deviations in flow rates were again evaluated in a histogram (Fig. 5).

In Fig. (6) the aldehyde production is shown in a contour plot as a function of time and stoichiometry. In Fig. (6a) the intended values (those set into the pumps) for time and stoichiometry were used, while in Fig. (6b) the values were calculated using the flow marker method. The high systematic deviation in stoichiometry is clearly visible here, and this has a significant impact on the interpretation of the results. When only set values are considered, the optimal aldehyde yield is reached at a stoichiometry of 2.5 and a reaction time of 2.5 s. However, when the accurate measured flow rates are used, the optimal reaction conditions are reached at a stoichiometry around 3.5 , at the same reaction time.

This result clearly demonstrates that when reaction optimization and screening in microreactors is considered one cannot rely on the set flow of the pumps involved. The significant average offsets are important observations, and can be explained by inaccuracy of the pump's mechanism and possible deviations in the diameters of the syringes used. Although it would be preferable to eliminate these inaccuracies, the offsets can also be taken into account when an experiment is set up. In either case, measurement of the flow
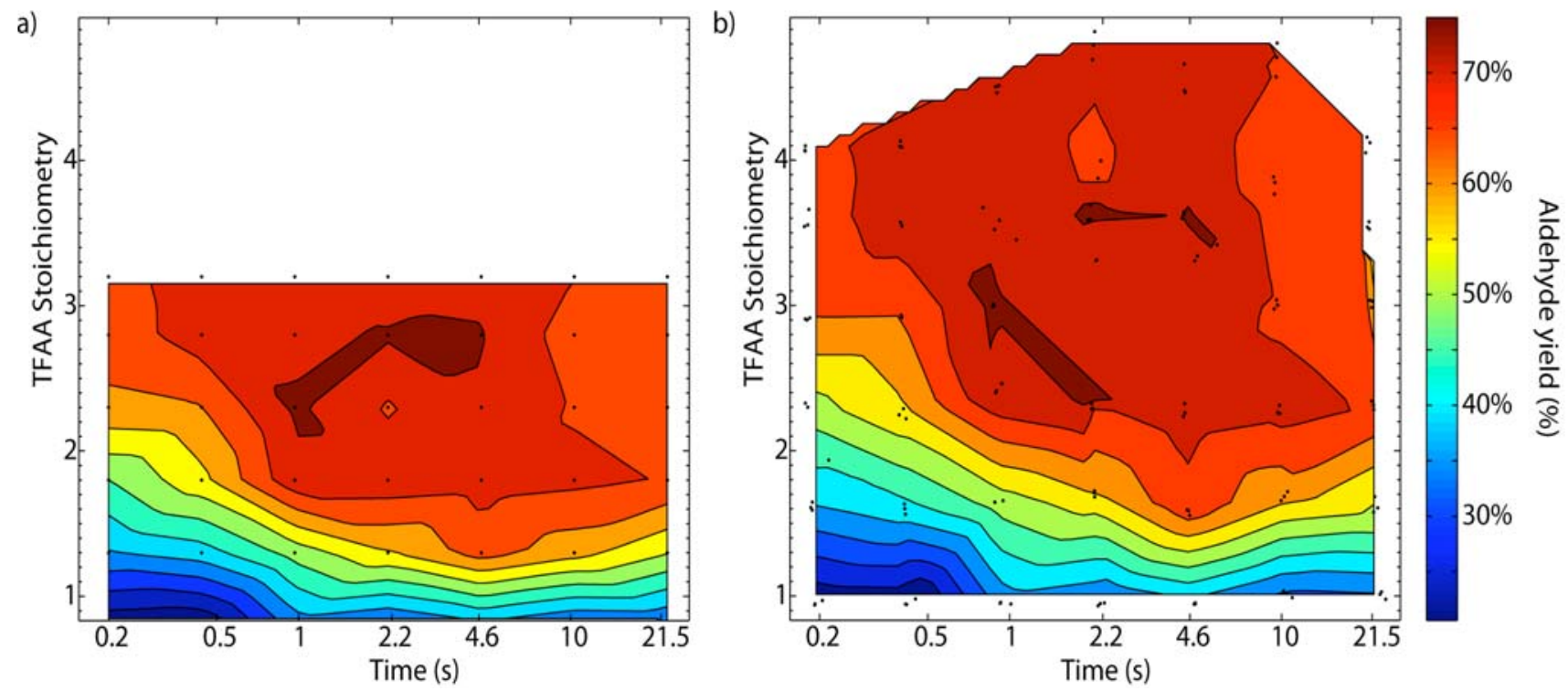

Fig. (6). Contour plots of Swern-Moffatt oxidation. The colored bands indicate the amount of aldehyde formed. (a): x-values and y-values are solely determined by the flow rates set into the pumps. (b): $x$-values and y-values are determined by actual flow measurements using the flow marker method. 
rates using flow markers during the entire course of the experiment allows for a more accurate end result of the optimization procedure.

\section{CONCLUSION}

A generally applicable method has been presented which can be used for flow rate analysis in microreactors for chemical synthesis using internal standards as flow markers. This method does not require additional instruments when chromatographic methods are used to monitor reaction products, since these methods can conveniently accommodate the extra measurements of flow markers. Although the new method in principle could be applied to a wide range of continuous flow synthesis applications, it is of a particular benefit when flow rates constitute an important experimental parameter, as is the case in reaction screening and determination of kinetics.

\section{ACKNOWLEDGEMENT}

This work was supported by the EUREGIO Rhine-Waal (Interreg IIIA) and the ministries of Economic affairs of the Netherlands and of North Rhine-Westphalia. Our project partners Fraunhofer IMS (Duisburg, Germany) and Wageningen University and Research Center (WUR, Wageningen, The Netherlands) are kindly acknowledged for fruitful discussions.

\section{SUPPLEMENTARY MATERIAL}

Supplementary material is available on the publishers Web site along with the published article.

\section{REFERENCES}

W. Ehrfeld, V. Hessel, and H. Löwe, "Microreactors - New Technology for Modern Chemistry", Weinheim, Wiley-VCH, pp. 5-12, 2000.

[2] K. Jänisch, V. Hessel, H. Löwe, and M. Baerns, "Chemistry in Microstructured Reactors", Angew. Chem. Int. Ed. Engl., vol. 43, pp. 406-446, 2004.

[3] P. Watts, and C. Wiles, "Recent advances in synthetic micro reaction technology", Chem. Commun., doi: 10.1039/b609428g, pp. 443-467, 2007.

[4] K. Geyer, J. D. C. Codee, and P. H. Seeberger, "Microreactors as tools for synthetic chemists - The chemists' round-bottomed flask of the 21st century?", Chem. Eur. J., vol. 12, pp. 8434-8442, 2006.

[5] B. P. Mason, K. E. Price, J. L. Steinbacher, A. R. Bogdan, and D. T. McQuade, "Greener approaches to organic synthesis using microreactor technology", Chem. Rev., vol. 107, pp. 2300-2318, 2007.

[6] H. Usutani, Y. Tomida, A. Nagaki, H. Okamoto, T. Nokami, and J. Yoshida, "Generation and Reactions of o-Bromophenyllithium without Benzyne formation using a microreactor", J. Am. Chem. Soc., vol. 129, pp. 3046-3047, 2007.

[7] M. S. Thomsen, P. Polt, and B. Nidetzky, "Development of a microfluidic immobilised enzyme reactor", Chem. Commun., vol. 24, pp. 2527-2529, 2007.

[8] R. Fortt, R. C. R. Wootton, and A. J. de Mello, "Continuous-flow generation of anhydrous diazonium species: Monolithic microfluidic reactors for the chemistry of unstable intermediates", Org. Process Res. Dev., vol. 7, pp. 762-768, 2003.

[9] C. Wiles, P. Watts, and S. J. Haswell, "The use of electroosmotic flow as a pumping mechanism for semi-preparative scale continuous flow synthesis", Chem. Commun., vol. 9, pp. 966-968, 2007.

[10] M. Fernandez-Suarez, S. Y. F. Wong, and B. H. Warrington, "Synthesis of a three-member array of cycloadducts in a glass microchip under pressure driven flow", Lab Chip, vol. 2, pp. 170-174, 2002.
[11] H. Hisamoto, T. Saito, M. Tokeshi, A. Hibara, and T. Kitamori, "Fast and high conversion phase-transfer synthesis exploiting the liquid-liquid interface formed in a microchannel chip", Chem. Commun., doi: 10.1039/b106494k, pp. 2662-2663, 2001.

[12] E. R. Murphy, J. R. Martinelli, N. Zaborenko, S. L. Buchwald, and K. F. Jensen, "Accelerating reactions with microreactors at elevated temperatures and pressures: Profiling aminocarbonylation reactions", Angew. Chem. Int. Ed. Engl., vol. 46, pp. 1734-1737, 2007.

[13] E. Garcia-Egido, V. Spikmans, S. Y. F. Wong, and B. H. Warrington, "Synthesis and analysis of combinatorial libraries performed in an automated micro reactor system", Lab Chip, vol. 3, pp. 73-76, 2003.

[14] K. Koch, R. F. J. van den Berg, P. J. Nieuwland, R. Wijtmans, M. G. Wubbolts, H. E. Schoemaker, F. P. J. T. Rutjes, and J. C. M. van Hest, "Enzymatic synthesis of optically pure cyanohydrins in microchannels using a crude cell lysate", Chem. Eng. J., vol. 135S, pp. S89-S92, 2008.

[15] K. Koch, R. F. J. van den Berg, P. J. Nieuwland, R. Wijtmans, H. E. Schoemaker, J. C. M. van Hest, and F. P. J. T. Rutjes, "Enzymatic enantioselective C-C-bond formation in microreactors", Biotechnol. Bioeng., vol. 99, pp. 1028-1033, 2008.

[16] K. Koch, B. J. A. van Weerdenburg, J. M. M. Verkade, P. J. Nieuwland, F. P. J. T. Rutjes, and J. C. M. van Hest, "Optimizing the deprotection of the amine protecting p-methoxyphenyl group in an automated microreactor platform", Org. Process Res. Dev., vol. 13, pp. 1003-1006, 2009.

[17] S. Devasenathipathy, J. G. Santiago, S. T. Wereley, C. D. Meinhart, and K. Takehara, "Particle imaging techniques for microfabricated fluidic systems", Exp. Fluids, vol. 34, pp. 504-514, 2003.

[18] F. Benito-Lopez, W. Verboom, M. Kakuta, J. G. E. Gardeniers, R. J. M. Egberink, E. R. Oosterbroek, A. van den Berg, and D. N. Reinhoudt, "Optical fiber-based on-line UV/Vis spectroscopic monitoring of chemical reaction kinetics under high pressure in a capillary microreactor", Chem. Commun., pp. 2857-2859, 2005.

[19] H. Lu, M. A. Schmidt, and K. F. Jensen, "Photochemical reactions and on-line UV detection in microfabricated reactors", Lab Chip, vol. 1, pp. 22-28, 2001.

[20] R. J. Jackman, T. M. Floyd, R. Ghodssi, M. A. Schmidt, and K. F. Jensen, "Microfluidic systems with on-line UV detection fabricated in photodefinable epoxy", J. Micromech. Microeng., vol. 11, pp. 263-269, 2001.

[21] R. Herzig-Marx, K. T. Queeney, R. J. Jackman, M. A. Schmidt, and K. F. Jensen, "Infrared spectroscopy for chemically specific sensing in silicon-based microreactors", Anal. Chem., vol. 76, pp. 6476-6483, 2004.

[22] S. A. Leung, R. F. Winkle, R. C. R. Wootton, and A. J. deMello, "A method for rapid reaction optimisation in continuous-flow microfluidic reactors using online Raman spectroscopic detection", Analyst, vol. 130, pp. 46-51, 2005

[23] P. D. I. Fletcher, S. J. Haswell, and X. L. Zhang, "Monitoring of chemical reactions within microreactors using an inverted Raman microscopic spectrometer", Electrophoresis, vol. 24, pp. 32393245, 2003.

[24] M. Tokeshi, T. Minagawa, K. Uchiyama, A. Hibara, K. Sato, H. Hisamoto, and T. Kitamori, "Continuous-flow chemical processing on a microchip by combining microunit operations and a multiphase flow network", Anal. Chem., vol. 74, pp. 1565-1571, 2002.

[25] A. Smirnova, K. Shimura, A. Hibara, M. A. Proskurnin, and T. Kitamori, "Application of a micro multiphase laminar flow on a microchip for extraction and determination of derivatized carbamate pesticides", Anal. Sci., vol. 23, pp. 103-107, 2007.

[26] A. Smirnova, K. Mawatari, A. Hibara, M. A. Proskurnin, and T. Kitamori, "Micro-multiphase laminar flows for the extraction and detection of carbaryl derivative", Anal. Chim. Acta, vol. 558, pp. 69-74, 2006.

[27] P. J. Nieuwland, K. Koch, N. van Harskamp, R. Wehrens, J. C. M. van Hest, and F. P. J. T. Rutjes, "Flash chemistry extensively optimized: high temperature Swern-Moffatt oxidation in an automated 
microreactor platform", Chem. Asian J., 2010, early view: DOI: 10.1002/asia.200900705.

[28] T. Kawaguchi, H. Miyata, K. Ataka, K. Mae, and J. Yoshida, "Room-temperature Swern oxidations by using a microscale flow system", Angew. Chem. Int. Ed. Engl., vol. 44, pp. 2413-2416, 2005.

[29] K. Omura, A. K. Sharma, and D. Swern, "Dimethylsulfoxidetrifluoroacetic anhydride - New reagent for oxidation of alcohols to carbonyls", J. Org. Chem., vol. 41, pp. 957-962, 1976.
[30] A. J. Mancuso, and D. Swern, "Activated dimethylsulfoxide - Useful reagents for synthesis", Synthesis, pp. 165-185, 1981.

[31] J. J. M. van der Linden, P. W. Hilberink, C. M. P. Kronenburg, and G. J. Kemperman, "Investigation of the Moffatt-Swern oxidation in a continuous flow micro reactor system", Org. Process Res. Dev., vol. 12, pp. 911-920, 2008.

(C) Nieuwland et al.; Licensee Bentham Open.

This is an open access article licensed under the terms of the Creative Commons Attribution Non-Commercial License (http://creativecommons.org/licenses/by-nc/3.0/) which permits unrestricted, non-commercial use, distribution and reproduction in any medium, provided the work is properly cited. 